\title{
Discussion on Full-coverage Technology of Campus Wireless Networks
}

\author{
Cheng Yin ${ }^{1, a}$, Guanglei Shan ${ }^{2, b}$, Hui Xi ${ }^{3, c}$, Wei Wang ${ }^{4, d}$ \\ ${ }^{1}$ Shandong Water Conservancy Vocational College,RizhaoChina \\ ${ }^{2}$ Shandong Water Conservancy Vocational College,RizhaoChina \\ ${ }^{3}$ Rizhao CICC, Rizhao China \\ ${ }^{4}$ Shandong Water Conservancy Vocational College,RizhaoChina

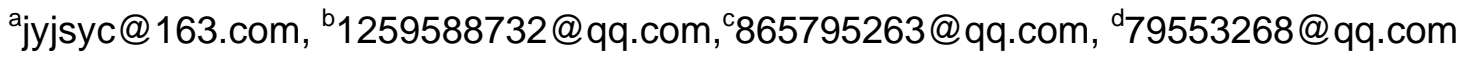

Keywords: Wireless networks, Network structure, No perception roaming

\begin{abstract}
This paper first introduces the propagation characteristics of wireless signals and the design principles of wireless networks. Through the analysis of campus wireless network needs, introduced the campus of several typical scenarios of wireless network coverage program. Through the analysis and comparison of the network structure, we can find a way to meet the needs of teachers and students to learn and work on the campus anytime, anywhere, and can realize the campus wireless full coverage without any way of roaming switching.
\end{abstract}

\section{Introduction}

Wireless local area network (WLAN) technology in the 20th century, 90 years gradually mature, both for the traditional wired network extension, in some environments can also replace the traditional wired network. Wireless LAN installation is simple and fast, reducing the number of laying pipes and threading work, and so on and the signal coverage is not subject to environmental restrictions. In the dynamic environment where frequent movement and change are needed, wireless LAN technology can better guarantee the flexibility and stability of information transmission.In the process of digital construction of the campus, the use of wireless network technology to further expand the coverage of the campus network, so that teachers and students to anytime, anywhere, convenient and efficient use of campus network, has become a school of digital campus construction of the necessary items.

\section{The propagation characteristics of wireless signals in space}

The propagation of wireless signals in space is related to many factors, including the transmission power of the AP, the gain of the antenna, the distance of the user, the interference signal of the space, the receiving sensitivity and so on.

Under vacuum conditions, the received signal received by the receiving antenna is only a fraction of the radiated power of the transmitting antenna during the transmission of the wireless signal. Most of the energy is diffused in other directions. The farther the working distance, the larger the ball area, the smaller the power intercepted at the receiving point, that is, the propagation loss increases. 
Vacuum wave propagation is the ideal way to spread radio waves. In practical applications, the transmission of wireless signals is much higher than that of vacuum due to interference from the environment.

The following are some common blocks of Wi-Fi signal loss values:

Table $1 \mathrm{Wi}$-Fi signal loss values for common barriers

\begin{tabular}{|c|c|}
\hline Barrier & Attenuation[db] \\
\hline Light wall & $5-8$ \\
\hline The middle wall & $10-15$ \\
\hline Heavy wall & $15-20$ \\
\hline Super heavy wall & $20-25$ \\
\hline Wooden wall & $5-6$ \\
\hline Glass (without metal oxide) & $3-4$ \\
\hline Glazing (containing metal oxide) & $5-10$ \\
\hline Ceiling (gypsum) & $10-15$ \\
\hline Ceiling (metal) & $20-30$ \\
\hline
\end{tabular}

For example, a 500mw AP, in the normal interference environment, to be the user to use the approximate acceptable space loss of $100 \mathrm{db}$. The signal to be $10 \mathrm{~m}$ distant users, in addition to the attenuation outside the normal distance, probably can be subjected to an additional attenuation of about 20db, i.e. up through the wall of the light blocking wall and two walls.

\section{Typical scenario scenario}

Typical coverage program on dormitory

Dormitory room is small, the number of partitions, more than the wall, the wireless environment is complex, staff-intensive, Internet staff focus on the network traffic demand is great. AP laying a panel-mounted AP in each room, and each AP does not cover more than one wall and is covered with a panel AP. And each AP out of four LAN port, for the PC Internet, to meet the different needs of the Internet (See Fig.1).

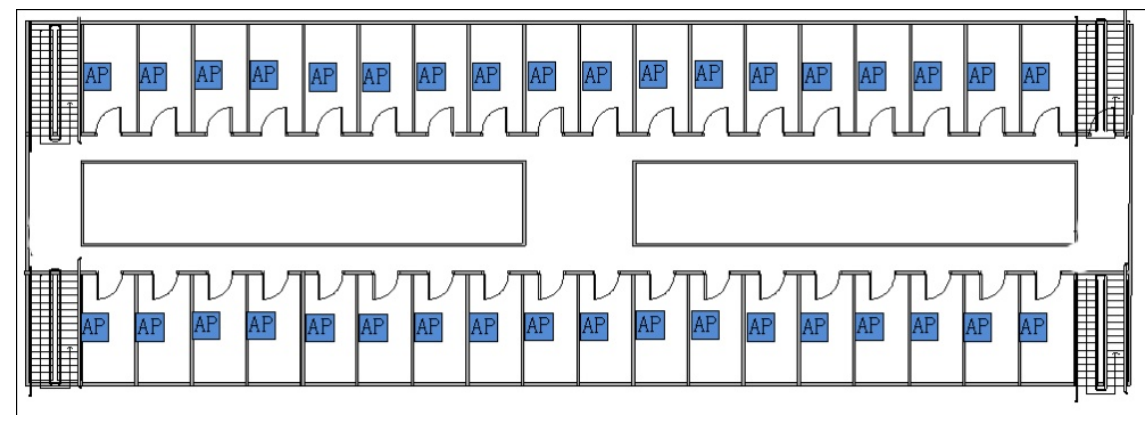

Fig.1 Dormitory coverage program

Typical coverage programon library

Library open floor plan, the complex structure of renovation, more people reading area, large flow requirements, taking into account the structure does not destroy the original decoration and routing issues, install dual ceiling AP (See Fig.2). 


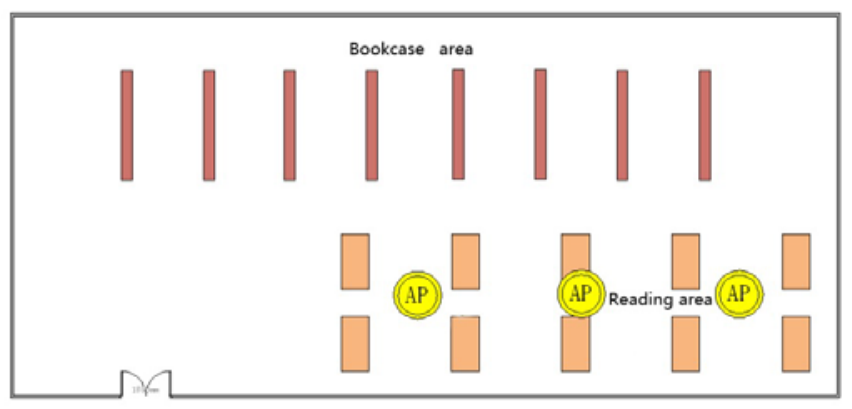

Fig.2 Library coverage program

Typical coverage programon classroom

100 seats within the classroom, dual-frequency ceiling AP coverage, not only can reduce interference between devices and can satisfy the coverage requirements. Large rooms should be based on the actual situation of 2 to 4 sets of dual-frequency ceiling AP, if the ceiling is too high, inconvenient construction, should be used to cover the wall-mounted AP (See Fig.3).

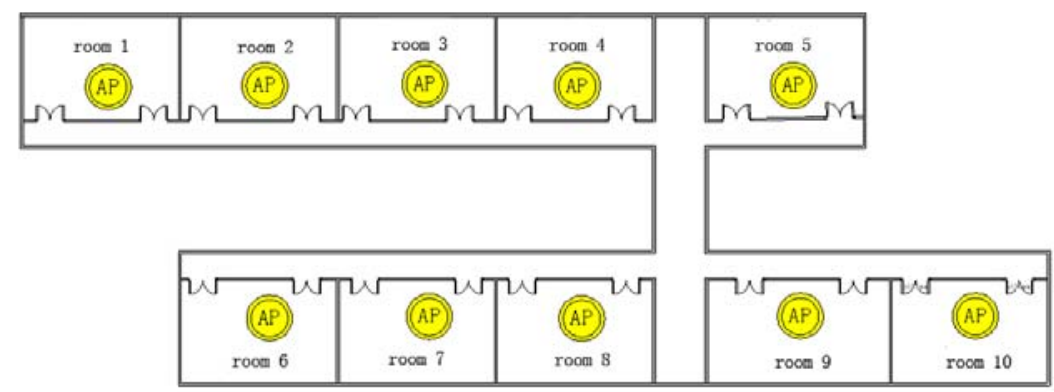

Fig. 3 Classroom coverage program

Outdoor typical coverage program

Outdoor wireless coverage of the target area, such as playground, dormitory and teaching building in the middle of the road, etc., need to complete the region at least $90 \%$ coverage.

1. Playground range, personnel-intensive, network access many people, but the Internet traffic needs are not high. Due to the installation location of the restrictions, the general use of outdoor orientation AP coverage. Also for the part of the stands, still use the ceiling AP or wall-mounted AP to cover (See Fig.4).

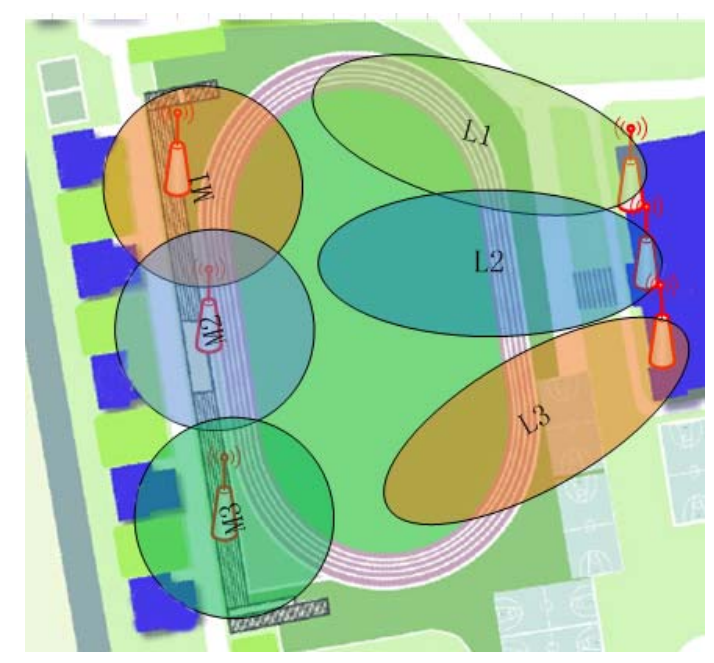

Fig.4 Playground coverage program 
2.Pavement area length, width, less Internet access, online roaming situation, the general use of outdoor orientation AP, along the road to cover.

High density user scenario typical coverage scheme

Large conference rooms, restaurants and other user-intensive places to the conference room, for example, the general length of $30 \mathrm{M}$, width of $24 \mathrm{M}$ or so, can accommodate between 300 to 500 people.

Ordinary AP access users in 30 to 40 people at the same time access, to deal with 300 to 500 places, only to increase the number of AP to meet the needs of access users.However, in a limited space, the increase in the number of APs is bound to introduce wireless and adjacent interference problems, reducing the overall performance of the device, can not meet the high-density users simultaneously access the bandwidth requirements.

We can solve the following two ways:

1) The use of high performance to meet more than 100 users at the same time access to the use of AP.

2) Each AP adaptive power reduction coverage, the same space frequency automatic planning. So that the inter-AP channels are independent of each other, and the power adaptation ensures that there is no intersection between the work items of the same channel. So that the performance of a single AP optimal, multi-device work at the same time doubled capacity,and multi-user access to the same time without congestion (See Fig.5).

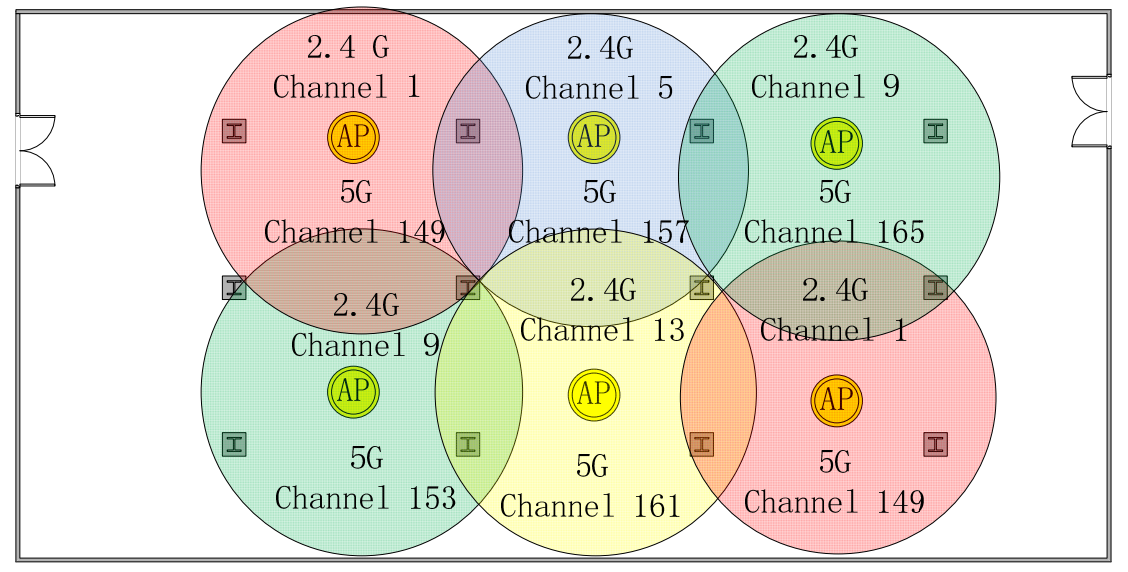

Fig.5 High density user scenario typical coverage scheme

\section{No sense roaming switch}

The wireless coverage of an AP is limited. A school requires hundreds of APs, and more than thousands of APs. If each AP is configured with a different SSID name, the user needs to manually reconnect each time through a room or through a place (See Fig.6). 


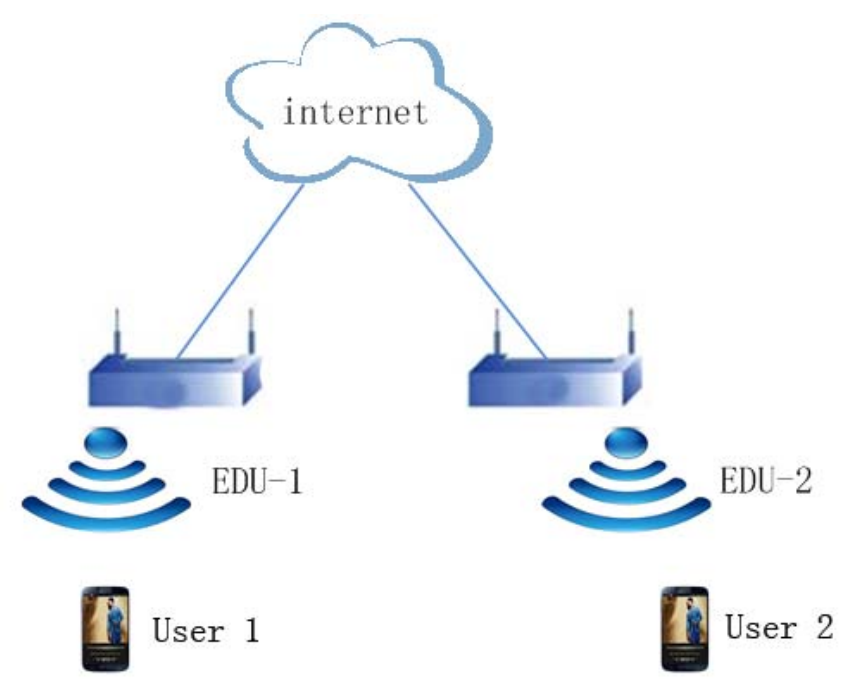

Fig.6 Networking of the wireless router

Each of the dormitories is equipped with a wireless router (with gateway function, direct access to the Internet).For example, the two names are: EDU-1 and EDU-2. Students from the room 1 to the room 2 must manually re-connect the EDU-2 and need to re-authenticate to access the Internet, which will result in a very poor user experience.

This network structure can save professional gateway equipment, cost savings, but can not group large network.It is applicable to shops, home and other small scenes.

If you have an AP (through an independent high-performance gateway to connect to the Internet) in both dormitories, the names are: EDU, students from room 1 to room 2, the system will automatically connect and authenticate, the user without any perception (See Fig.7).

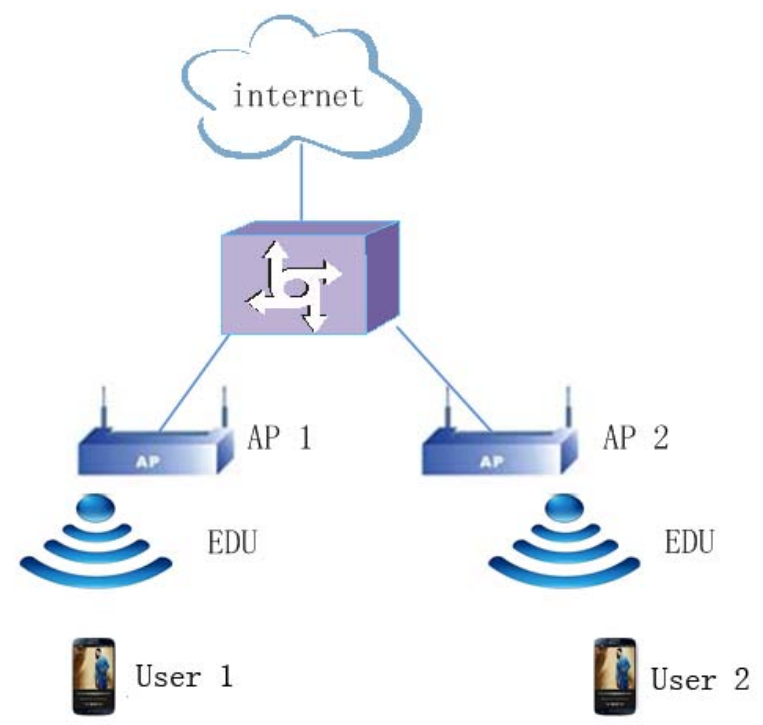

Fig.7 AP networking structure

The user experience of this networking structure is good, as long as the first association certified teachers and students, the school can use seamless roaming. But need to invest in professional gateway equipment, high cost, suitable for schools, enterprises and other large scenes.

At present, the full coverage of the campus wireless network has become an important part of the construction of intelligent campus. AP network structure has become the campus wireless network construction of the primary choice. To achieve no sense of roaming switch, first unified 
SSID, to ensure that the user terminal can be automatically connected after a link; At the same time, the bidirectional switching control algorithm is adopted, and the local AC initiates the switching process according to the terminal signal strength measured by several adjacent APs. Terminal side APP control switching algorithm. The terminal side APP initiates the switching process according to the strength of the AP signal strength of several neighboring systems measured by the terminal.

The system uses the cloud platform to record user authentication status. All APs are managed by the same cloud platform, broadcast the same SSID and in the same service VLAN, the cloud platform to save the user's SSID, MAC address, IP address, wireless encryption and authentication information. When a roaming occurs, the cloud platform from the same MAC address to determine the roaming, will keep the business attributes unchanged, no address changes and re-certification and so on. User terminal data through the gateway to the cloud platform, the cloud platform record with the authentication state, so that the same hot group certification once, the user after the cross-hot certification status unchanged, to ensure that users do not need to repeat the certification (See Fig.8).
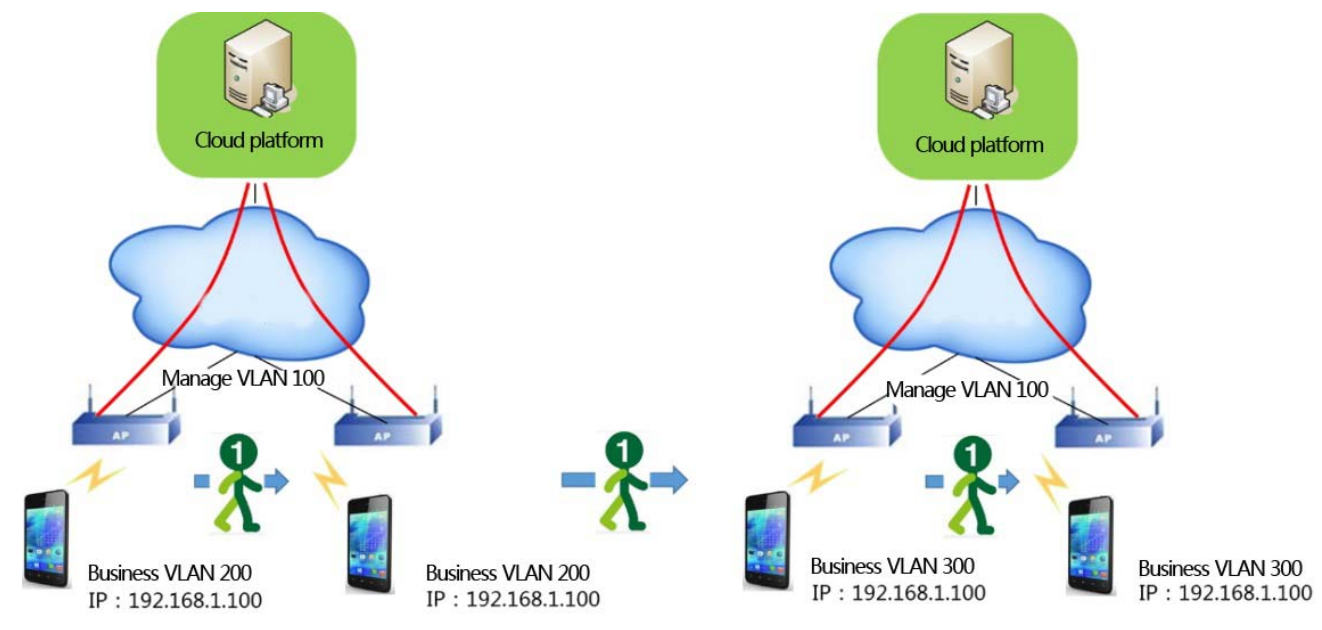

Fig.8 Cloud platform record user authentication status

\section{References:}

[1]Li Lianye, GuoChunfang, Xiang Dongming, Wireless network and its application technology, Tsinghua University Press Publication Date: 2004/6/1

[2]GaoChunyang,Bai Liang, Sun Ping.Study on Wireless Network Construction in Colleges and Universities [J]. Computer Knowledge and Technology, 2013.9

[3]Wei Zhang: Research and Design of Security Architecture of Wireless Campus Network [D] Yunnan University, 2014 